\title{
Estudo comparativo de ferros fundidos nodulares temperados e austemperados
}

\section{Comparative study of quenched and austempered ductile cast irons}

Paulo Henrique Sanchez Cardoso

Prof. Dr. Escola de Engenharia, FURG Rua Alfredo Huch, 475

96201-900, Rio Grande, RS, Brasil paulocardoso@furg.br

Charles Leonardo Israel

Prof. Dr. Faculdade de Engenharia e Arquitetura, UPF

BR $285 \mathrm{~S} / \mathrm{N}$

99052-900, Passo Fundo, RS, Brasil

israel@upf.br

Telmo Roberto Strohaecker

Prof. Dr. Departamento de Metalurgia, UFRGS

Av. Osvaldo Aranha 99/610

90035-190, Porto Alegre, RS, Brasil telmo@demet.ufrgs.br

\section{Resumo}

Este trabalho busca avaliar propriedades mecânicas de três tipos de ferros fundidos, relacionando-a com fatores metalúrgicos das amostras. Em relação a propriedades mecânicas, avaliou-se a resistência ao impacto de um ferro fundido nodular austemperado - FFNA - com 0,4\% de Mo (temperaturas de austêmpera em 260 e $300^{\circ} \mathrm{C}$ ) e de um ferro fundido nodular temperado em sais e revenido a $200^{\circ} \mathrm{C}$ FFNTR. Adicionalmente, foram verificados os limites de resistência à tração e seus valores de dureza, bem como, foram realizadas análises fractográficas nos corpos de prova ensaiados. Em termos metalúrgicos, foi verificada a quantidade de nódulos nas amostras e o percentual de austenita retida. Para os testes de impacto, o ferro fundido nodular austemperado em $300^{\circ} \mathrm{C}$ apresentou a maior resistência, a qual foi relacionada com a sua elevada quantidade de austenita retida (aproximadamente $21 \%)$.

Palavras-chave: ferro fundido nodular austemperado, propriedades mecânicas, austenita retida.

\section{Abstract}

This work aims to evaluate the mechanical properties of three different materials, relating the tests results with the metallurgical features of the samples. The first and the second material were an austempered ductile cast iron (austempering temperatures of 260 and $300^{\circ} \mathrm{C}$, respectively). The third material was a quenched and tempered ductile cast iron. Impact, tension and hardness tests were employed in the mechanical characterization of the materials. In the metallurgical characterization, optical microscopy and image analysis were employed. It was observed that in the impact tests, the austempered ductile iron in the temperature range of $300^{\circ} \mathrm{C}$ was the material with higher toughness. In this material, low hardness allied to the high retained austenite level $(21 \%)$, were the main factors acting in the higher impact resistance of the $300^{\circ} \mathrm{C}$ austempered cast iron.

Key words: ADI, mechanical properties, retained austenite. 


\section{Introdução}

Incontáveis são as vantagens da utilização dos ferros fundidos na indústria, principalmente na manufatura em larga escala de componentes com geometria complexa. Contudo, os principais problemas na utilização dos ferros fundidos sempre estiveram relacionados com a baixa tenacidade e ductilidade destes materiais quando comparadas com os aços (Hsu e Chuang, 2001). Nesta lacuna se apresenta o ferro fundido nodular austemperado (FFNA) ou Austempered Ductile Iron (ADI), um material com as vantagens dos ferros fundidos, como fundibilidade e produtividade, e que apresenta ainda excelentes valores de resistência mecânica e tenacidade. Comparando com os ferros fundidos nodulares tradicionais, os austemperados permitem, para o mesmo nível de ductilidade, mais que duplicar a resistência à tração (Santos e Branco, 1989). Para o presente trabalho, utilizou-se um ferro fundido nodular temperado e revenido (FFNTR), em função de custo de fabricação e de elevada dureza, e dois tipos de ferros fundidos austemperados (FFNA). Estes dois tipos basearam-se em temperaturas de austêmpera diferentes (260 e $300^{\circ} \mathrm{C}$ ), sendo que nestes materiais acrescentou-se Mo como elemento de liga. O molibdênio é um importante agente endurecedor adicionado ao ferro nodular. Entretanto, pode segregar na fronteira das células durante a solidificação, formando carbonetos e limitando, muitas vezes, sua adição a 0,3\% (Trudel e Gagné, 1997). Para peças de menor velocidade de solidificação, peças mais espessas, podem ser empregados teores de até $0,8 \%$ de Mo sem que ocorra a formação de carbonetos. Esse elemento também pode ser empregado para aumentar a resistência de ferros fundidos nodulares ferríticos obtidos após recozimento. Todavia, esse uso pode não ser aconselhável no caso de se necessitar de elevada resistência ao impacto (Yescas-González, 2001). A quantidade de grafita também é um importante fator a ser considerado. Com o aumento do número de nódulos, há uma redução do valor de energia absorvida no impacto. Isso pode ser explicado pelo mecanismo de iniciação e propagação de trincas, o qual consiste em deformação plástica localizada nas regiões de matriz entre nódulos, resultando em microestriç̧ão, formação e coalescimento de microcavidades. Formamse assim microtrincas, as quais se ligam à trinca principal, reduzindo a resistência ao impacto. Esses fenômenos ocorrem mais facilmente à medida que aumenta o número de nódulos, em função do menor espaçamento entre eles (Valdes et al., 2009).

\section{Materiais e Métodos}

As corridas que geraram o ferro fundido nodular destinado a tempera e revenido (FFNTR) e o ferro fundido nodular destinado a austêmprera nas temperaturas de 260 e $300^{\circ} \mathrm{C}$ (FFNA) foram produzidas em forno à indução Inductotherm, capacidade nominal de $320 \mathrm{~kg}, 950 \mathrm{~Hz}, 150 \mathrm{kVA}$ e $450 \mathrm{~V}$. Para ambas as corridas a carga foi constituída de $92 \%$ de ferro gusa e $8 \%$ de sucata de aço. O tratamento de nodulização foi realizado na panela com $1,5 \%$ de FeSiMg ( $46 \%$ de Si e $6,2 \%$ de $\mathrm{Mg}$ ) e a inoculação no jato do metal líquido, com 0,5\% de FeSi75. A temperatura de fusão foi de $1540^{\circ} \mathrm{C}$. A diferença existente entre o FFN e o FFNA foi a adição de $0,25 \%$ de FeMo (60\%Mo) no FFNA, aumentando, assim, o teor de Mo na liga e, conseqüentemente, a sua temperabilidade. $O$ metal obtido em cada fusão foi vazado em coquilha (para a obtenção da amostra para análise química). Para ambas as corridas de ferro fundido nodular (FFNs) foram fundidos blocos em "Y", de $25 \mathrm{~mm}$ de espessura, de onde foram retirados corpos-de-prova para a realização dos ensaios de tração e de impacto (Hafiz, 2003). Os corpos-de-prova foram usinados e, em seguida, 
realizados os tratamentos térmicos. O tratamento térmico do ferro fundido nodular austemperado baseou-se em pré-aquecimento a $450^{\circ} \mathrm{C}$ por aproximadamente $2 \mathrm{~h}$, austenitização do material a $900^{\circ} \mathrm{C}$ durante $2 \mathrm{~h}$ em banho de sal e posterior austêmpera durante $2 \mathrm{~h}$ em temperaturas de $260^{\circ} \mathrm{C}$ para um lote, e a $300^{\circ} \mathrm{C}$ para outro lote, ambas em banho de sais. Para o tratamento térmico do ferro fundido nodular temperado e revenido utilizou-se de pré-aquecimento a $450^{\circ} \mathrm{C}$ por aproximadamente $2 \mathrm{~h}$, austenitização do material a $900^{\circ} \mathrm{C}$ durante $2 \mathrm{~h}$ em banho de sal, posterior têmpera em banho de sais e revenimento subseqüente durante $2 \mathrm{~h}$ a $200^{\circ} \mathrm{C}$. Os corpos-de-prova para ensaios de tração foram usinados e, posteriormente, sofreram tratamento térmico, sendo em número de três para cada condição. Os ensaios foram realizados segundo a Norma ASTM E8M-00b com o diâmetro da parte útil de 12,5 mm. Para os ensaios de dureza foi utilizada a escala Brinell, com esfera de $10 \mathrm{~mm}$ de diâmetro e carga de $3000 \mathrm{kgf}$. Os ensaios foram realizados segundo a Norma ASTM E10-01 em número de cinco para cada condição. Os ensaios Charpy foram realizados à temperatura ambiente, $21^{\circ} \mathrm{C}$, e obedeceram à norma ASTM E23-01 em corpos de prova com entalhe.

\section{Resultados e Discussão}

A Tabela 1 apresenta a composição química dos materiais obtidos, juntamente com o valor de carbono equivalente.

Tabela 1: Composição química dos materiais estudados (\% em peso).

\begin{tabular}{|c|c|c|c|c|c|c|c|c|}
\cline { 2 - 9 } \multicolumn{1}{c|}{} & $\mathrm{C}$ & $\mathrm{Si}$ & $\mathrm{Mn}$ & Mo & $\mathrm{P}$ & $\mathrm{Mg}$ & $\mathrm{S}$ & $\mathrm{CE}$ \\
\hline FFNTR & 3,81 & 2,30 & 0,21 & 0,08 & 0,05 & 0,04 & 0,01 & 4,6 \\
\hline FFNA & 3,72 & 2,39 & 0,21 & 0,42 & 0,05 & 0,04 & 0,01 & 4,5 \\
\hline
\end{tabular}

Um dos fatores a se considerar na produção dos ferros fundidos é o teor de carbono equivalente (CE). Para a fabricação de FFNs geralmente se recomenda o emprego de ligas de composição eutética ou hipereutética, com CE situado entre 4,3 e 4,7\%. Os teores de carbono mais freqüentemente empregados situam-se entre 3,4 e 3,8\% e os de silício, entre 2,1 e 2,8\%. Nessas condições, obtêm-se, geralmente, nódulos de grafita mais perfeitos (Hupalo et al., 2006).

A fórmula para obtenção do carbono equivalente está apresentada na Equação 1.

$$
\% C E=\% C+\frac{(\% S i+\% P)}{3}
$$

O carbono equivalente é um índice que indica a combinação efetiva dos elementos silício e fósforo em termos de influência com o carbono, conforme indicado na Equação 1. Com o aumento do CE, o limite de resistência à tração diminui, o mesmo acontecendo com a dureza (Yescas-González, 2001).

A Figura 1 apresenta as micrografias representativas das amostras. 

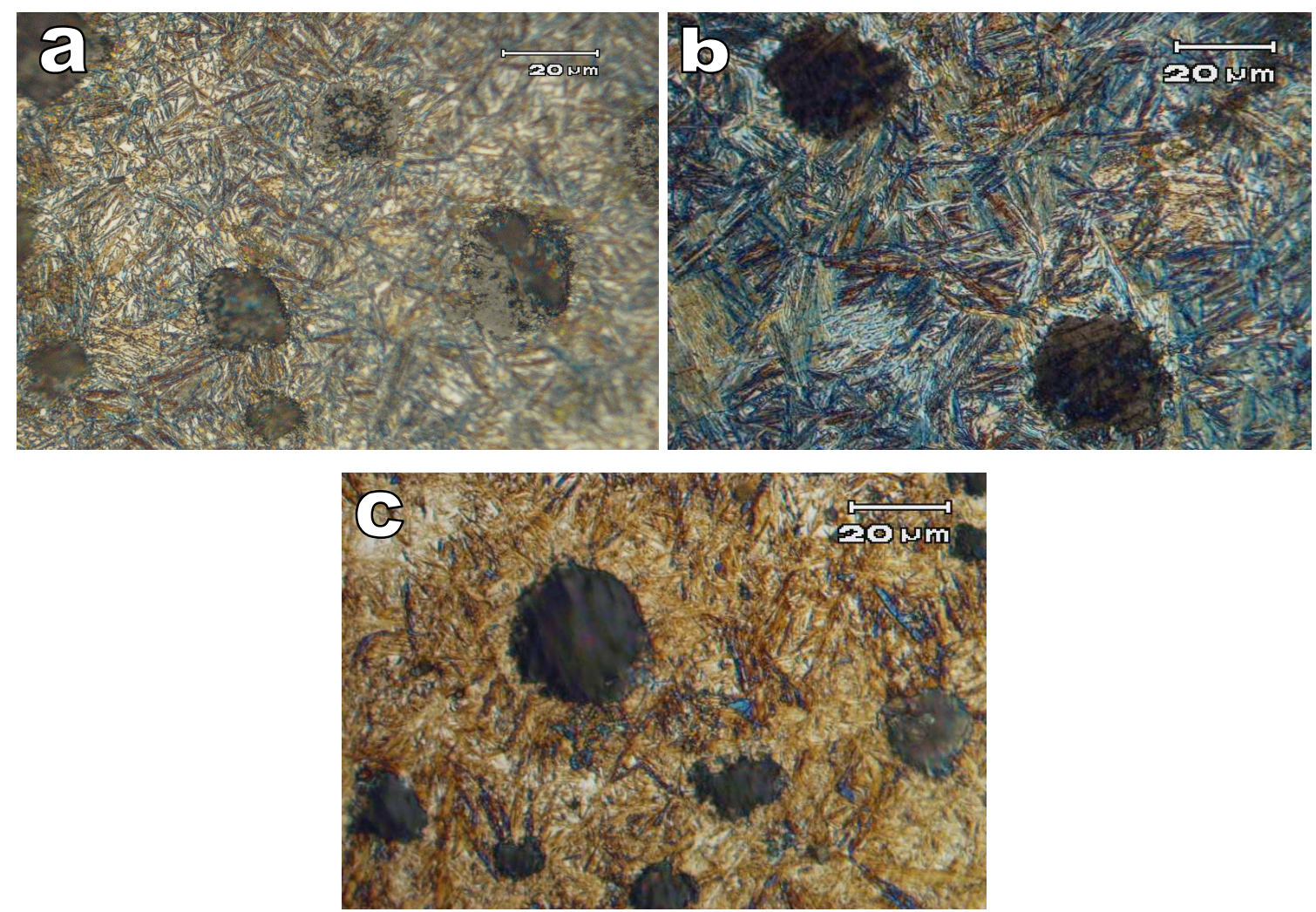

Figura 1: Metalografias representativas das amostras: (a) FFNA $260^{\circ} \mathrm{C}$, (b) FFNA $300^{\circ} \mathrm{C}$ e (C) FFNTR.

A contagem dos aspectos pertinentes as imagens as imagens foi realizado com o auxilio do software "Image Tool", e os valores encontrados são apresentados na Tabela 2.

Tabela 2: Microconstituintes dos ferros fundidos nodulares.

\begin{tabular}{|c|c|c|c|}
\hline Microestrutura & FFNA260 ${ }^{\circ} \mathrm{C}$ & FFNA300 ${ }^{\circ} \mathrm{C}$ & FFNTR \\
\hline Nódulos/mm ${ }^{2}$ & $283-339$ & $249-305$ & $346-462$ \\
\hline $\begin{array}{c}\text { Tamanho da Grafita } \\
\text { (ASTM A 247) }\end{array}$ & $6-7$ & $6-7$ & $7-8$ \\
\hline Grau de nodularização & $>90 \%$ & $>90 \%$ & $>90 \%$ \\
\hline Matriz & $\begin{array}{c}\text { Ferrita acicular } 88 \% \\
\text { Austenita retida 12\% }\end{array}$ & $\begin{array}{c}\text { Ferrita acicular } 79 \% \\
\text { Austenita retida } 21 \%\end{array}$ & $\begin{array}{c}\text { Martensita revenida } \\
\text { Austenita retida }<5 \%\end{array}$ \\
\hline
\end{tabular}

A diferença observada da quantidade de nódulos $/ \mathrm{mm}^{2}$ entre o FFNA e FFNTR é relacionada com a presença de molibdênio nos FFNA, por ser este um elemento que significativamente reduz a eficiência do inoculante (Hanc e Binczyk, 2008). O percentual de austenita retida encontrado na matriz dos FFNAs é similar ao encontrado na literatura (Yescas-González, 2001), de aproximadamente, $12 \%$ para o FFNA260 ${ }^{\circ} \mathrm{C}$ e de $21 \%$ para o FFNA $300^{\circ} \mathrm{C}$.

A Tabela 3 apresenta os resultados de dureza e dos ensaios de tração realizados nos corpos de prova (S - desvio padrão). 
Tabela 3: Propriedades de dureza e resistência a tração dos ferros fundidos nodulares (S - desvio padrão).

\begin{tabular}{|c|c|c|c|c|c|c|}
\hline \multirow{2}{*}{$\begin{array}{c}\text { Propriedades } \\
\text { Mecânicas }\end{array}$} & \multicolumn{2}{|c|}{ FFNA260 } & \multicolumn{2}{c|}{ FFNA300 $00^{\circ} \mathrm{C}$} & \multicolumn{2}{c|}{ FFNTR } \\
\cline { 2 - 7 } & Média & $\mathrm{S}$ & Média & $\mathrm{S}$ & Média & $\mathrm{S}$ \\
\hline Dureza HB & 414 & 7,0 & 397 & 4,0 & 549 & 5,0 \\
\hline $\begin{array}{c}\text { Resistência a Tração } \\
\text { (MPa) }\end{array}$ & $1.291,3$ & 13,5 & $1.189,8$ & 59,6 & $1.150,6$ & 67 \\
\hline
\end{tabular}

Observa-se na Tabela 3 que o FFNA $260^{\circ} \mathrm{C}$ apresentou dureza e resistência à tração maiores que o FFNA $300^{\circ} \mathrm{C}$, em razão da sua temperatura de austêmpera ser inferior. O valor de resistência a tração do FFNA $260^{\circ} \mathrm{C}$ foi em torno de $8 \%$ superior ao do FFNA $300^{\circ} \mathrm{C}$, tomando o maior como referência. Em uma comparação do FFNA $260^{\circ} \mathrm{C}$ com o FFNTR, o valor de resistência a tração do $\mathrm{FFNA} 260^{\circ} \mathrm{C}$ passou a ser em torno de $10 \%$ superior ao FFNTR, mas neste caso não pode ser feita a mesma relação com a dureza que foi feita anteriormente, pois o valor de dureza do FFNTR foi maior que o do FFNA260 ${ }^{\circ} \mathrm{C}$. Já na comparação entre o FFNA $300^{\circ} \mathrm{C}$ e o FFNTR, ambos apresentam limite de resistência à tração similares, embora o FFNA300 ${ }^{\circ} \mathrm{C}$ apresente dureza bastante inferior ao FFNTR. As observações indicam alta fragilidade do material FFNTR, pois apresenta a maior dureza dos três e, ao mesmo tempo, a menor resistência a tração dos três.

A Tabela 4 apresenta os resultados dos ensaios de impacto para corpos de prova sem e com entalhe. Primeiramente, em função da alta dureza dos corpos de prova, os ensaios de impacto foram feitos em corpos de prova sem entalhe, o que facilitaria o processo de usinagem. Também, em função de serem esperados baixos valores de energia de impacto absorvida, optou-se pelo uso de corpos de prova sem entalhe para que os valores de energia absorvida fossem mais altos. Após a realização dos ensaios, foram verificadas altas dispersões de resultados para os corpos de prova $\left(67,2 \%\right.$ da média para o FFNA260 ${ }^{\circ} \mathrm{C}$, $43,8 \%$ da média para o FFNA $260^{\circ} \mathrm{C}$ e $41 \%$ da média para o FFNTR). Em uma segunda etapa, os ensaios foram refeitos com corpos de prova entalhados, diminuindo assim a dispersão de resultados em relação a média dos corpos de prova, sendo isto devido ao direcionamento da fratura causado pela concentração de tensões relacionada com a presença do entalhe (dispersões de $1,7 \%$ da média para o FFNA260 ${ }^{\circ} \mathrm{C}, 2,3 \%$ da média para o FFNA $260^{\circ} \mathrm{C}$ e $4,6 \%$ da média para o FFNTR). Os valores indicados na média e S (desvio padrão) são resultado de cinco corpos de prova ensaiados para cada condição.

Tabela 4: Resultados do ensaio de impacto em corpos-de-prova com e sem entalhe (S - desvio padrão).

\begin{tabular}{|c|c|c|c|c|c|c|}
\cline { 2 - 7 } \multicolumn{1}{c|}{} & \multicolumn{2}{c|}{ FFNA260 $260^{\circ} \mathrm{C}$} & \multicolumn{2}{c|}{ FFNA300 0 C } & \multicolumn{2}{c|}{ FFNTR } \\
\cline { 2 - 7 } \multicolumn{1}{c|}{} & Média & $\mathrm{S}$ & Média & $\mathrm{S}$ & Média & $\mathrm{S}$ \\
\hline Sem entalhe & $39,1 \mathrm{~J}$ & 26,3 & $67,2 \mathrm{~J}$ & 29,4 & $35,1 \mathrm{~J}$ & 14,4 \\
\hline Com entalhe & $6,0 \mathrm{~J}$ & 0,1 & $8,6 \mathrm{~J}$ & 0,2 & $4,3 \mathrm{~J}$ & 0,2 \\
\hline
\end{tabular}

Como pode ser observado na tabela 4, a tendência de resultados obtida nos ensaios de impacto é a mesma, tanto para corpos de prova sem quanto com entalhe, isto é, maiores valores de energia absorvida obtidos para o FFNA $300^{\circ} \mathrm{C}$, seguida do FFNA $260^{\circ} \mathrm{C}$ e do FFNTR.

Tais observações podem ser explicadas, principalmente, pelo fato de a energia absorvida em um ensaio de impacto ser inversamente proporcional a dureza das amostras ensaiadas (Ahmadabadi et al., 
1999), sendo que o FFNA $300^{\circ} \mathrm{C}$ apresentou dureza de $397 \mathrm{HB}$, o FFNA $260^{\circ} \mathrm{C}$ de $414 \mathrm{HB}$ e FFNTR de $549 \mathrm{HB}$ (Tabela 3).

Ainda em relação à Tabela 4, menciona-se que o material FFNTR possui a maior quantidade de nódulos de grafita por $\mathrm{mm}^{2}$ das três amostras, bem como maior tamanho médio de grafita (presença de Mo nas amostras de FFNA - redutor de eficiência de inoculante). A maior quantidade de nódulos e o maior tamanho médio de grafita (Tabela 2) levam a baixos valores de energia absorvida, pois existe menor distância relativa entre os nódulos, favorecendo o coalescimento dos alvéolos e diminuindo a resistência a propagação da trinca (Putatunda, 2001).

A Figura 2 apresenta o aspecto de fratura dos corpos de prova submetidos aos ensaios de impacto (corpos de prova sem entalhe).
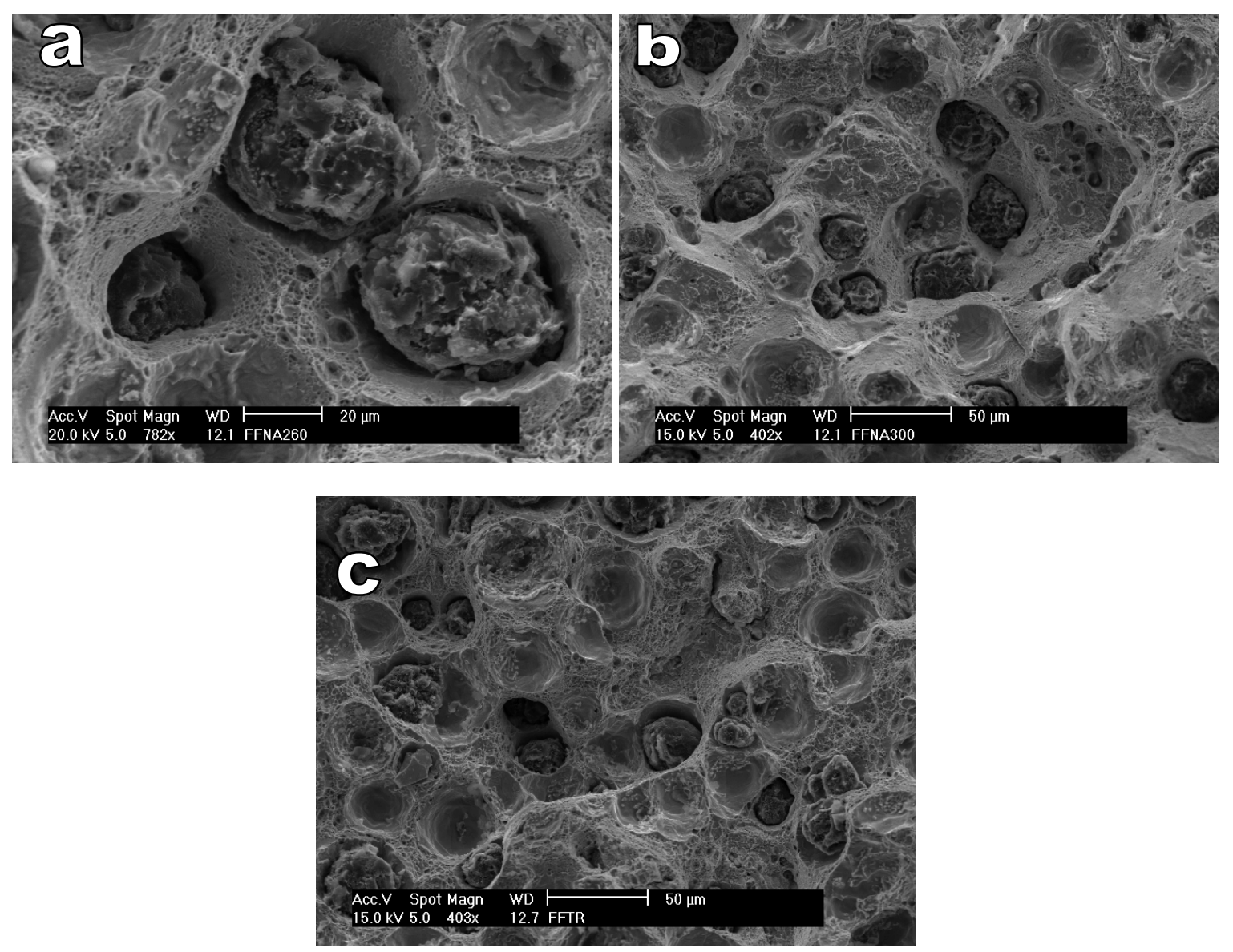

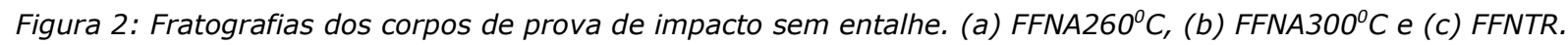

A Figura 3 apresenta o aspecto de fratura dos corpos de prova submetidos aos ensaios de impacto (corpos de prova com entalhe). 

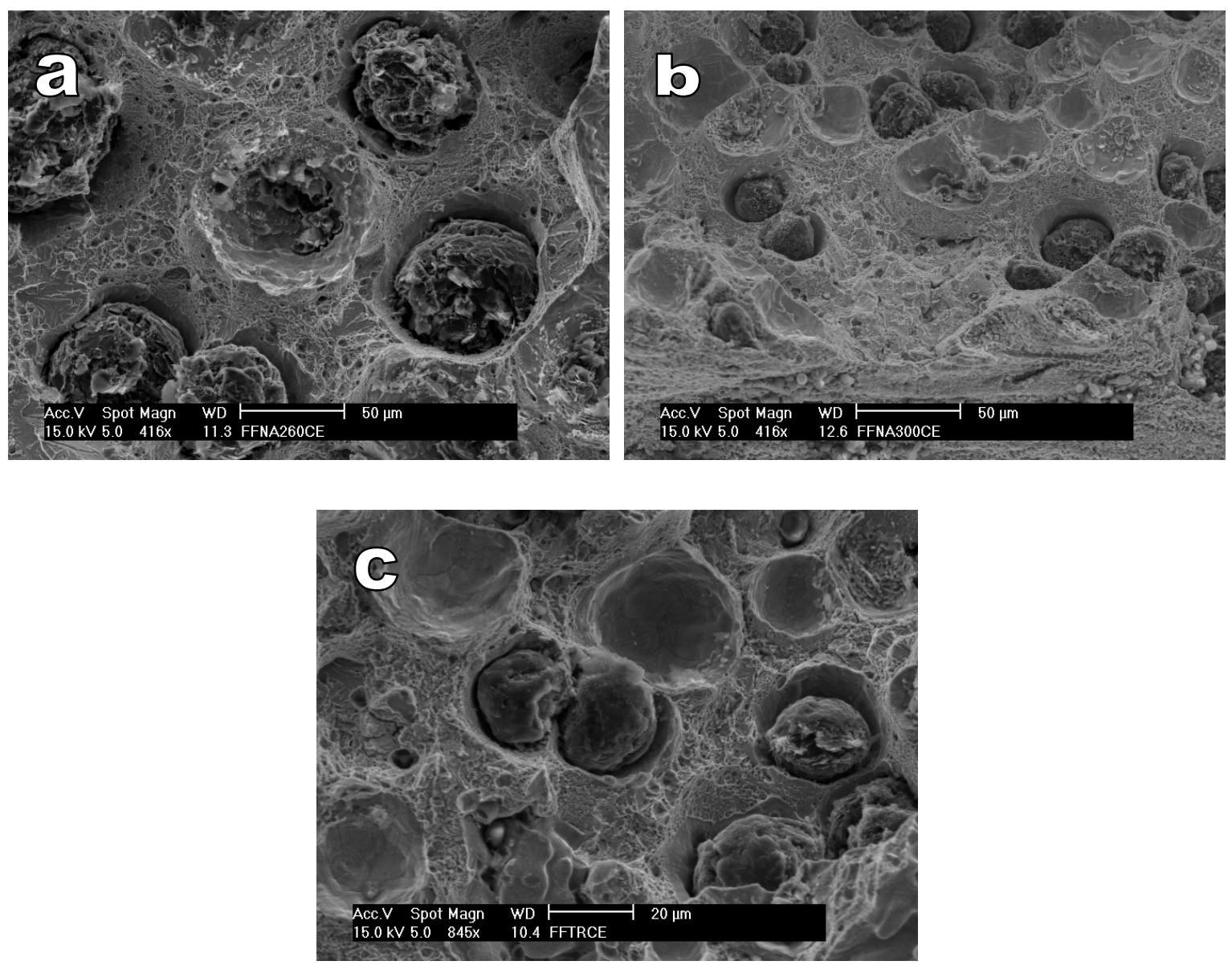

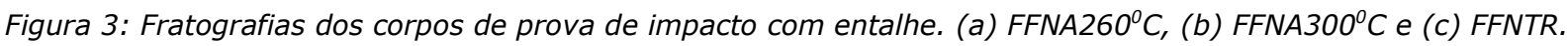

De um modo geral, todas as amostras de ferro fundido nodular apresentaram deformação ao redor dos nódulos de grafita, com ocorrência de "dimples" mais pronunciados nas amostras com maior energia absorvida no ensaio de impacto (FFNA $300^{\circ} \mathrm{C}$ ). Ainda, para todas as amostras ensaiadas, verificou-se decoesão dos nódulos de grafita de suas cavidades durante a fratura. Não foi observada fratura característica de clivagem, a qual, de certa forma, esperava-se encontrar no FFNTR, em razão da sua microestrutura martensítica. Um dos fatores que podem ter influenciado na presença predominantemente de dimples nas amostras foi o baixo teor de fósforo e silício encontrados - fragilizantes típicos (Francucci et al., 2008).

\section{Conclusão}

Conclui-se que o processo de produção do material foi correto em termos de geração de amostras de ferro fundido nodular com valores esperados de carbono equivalente e de grau de nodularização. Ainda, os tratamentos térmicos empregados nas amostras geraram as diferenças estruturais necessárias para que as comparações pertinentes pudessem ser feitas.

Para as amostras produzidas, os valores de carbono equivalente foram muito semelhantes, assim todas as mudanças de comportamento das amostras em termos de resistência a tração e energia de impacto são relacionadas com aspectos microestruturais provenientes dos tratamentos térmicos empregados. 
A temperatura de austêmpera determina a quantidade de austenita retida. Para uma mesma temperatura de austenitização, temperaturas de austêmpera mais altas irão gerar maior quantidade de austenita retida.

Mesmo para corpos de prova de elevada dureza, os ensaios de impacto devem ser feitos com corpos de prova entalhados, para que não seja obtida uma grande dispersão de resultados de energia absorvida. 0 entalhe funciona como um concentrador de tensões e direciona a propagação da trinca.

Dos três materiais estudados, o FFNTR foi o que apresentou maior fragilidade, possuindo a maior dureza, a menor resistência a tração e os menores valores de energia de impacto. Basicamente, tal fator é associado a alta dureza e a menor distância relativa entre os nódulos de grafita.

Em relação a tenacidade, o $F F N A 300^{\circ} \mathrm{C}$ foi considerado o melhor dos três. Obteve a menor dureza entre os três materiais e a maior energia de impacto. Em termos de resistência a tração, obteve um limite em torno de $8 \%$ inferior ao FFNA $260^{\circ} \mathrm{C}$, mas uma média de energia absorvida em torno de $40 \%$ superior ao mesmo FFNA $260^{\circ} \mathrm{C}$. Acredita-se que, para o caso do ensaio de tração, por ser um ensaio estático, a quantidade de austenita retida não possui grande influência, pois ambos os materiais teriam toda a quantidade de austenita retida transformada em martensita por deformação ao final do ensaio. Assim, maiores valores de dureza implicam em maior resistência a tração. Já para ensaios dinâmicos, nem toda a austenita retida se transforma por deformação, assim quanto maior o percentual de austenita retida, maior a energia absorvida, o que é o caso do $\operatorname{FFNA} 300^{\circ} \mathrm{C}$, que possui maior quantidade de austenita em comparação com o FFNA $260^{\circ} \mathrm{C}(21$ em relação a $12 \%)$.

As propriedades de impacto das ligas avaliadas são coerentes com as microestruturas avaliadas, entretanto, para os FFNAs, um pouco inferiores a alguns trabalhos consultados, provavelmente resultante do elevado teor de molibdênio utilizado como elemento de liga (Nofal e Jekova, 2009; Ravishankar et al., 2008).

\section{Referências}

AHMADABADI, N.M.; GHASEMI, H.M.; OSIA, M. 1999. Effects of successive austempering on the tribological behavior of ductile cast iron. Wear, 231(2):293-300. http://dx.doi.org/10.1016/S00431648(99)00163-5

FRANCUCCI, G.; SIKORA, J.; DOMMARCO, R. 2008. Abrasion resistance of ductile iron austempered by the two-step process. Materials Science and Engineering $A$, 485(1-2):46-54. http://dx.doi.org/10.1016/j.msea.2007.07.081

HAFIZ, M. 2003. Mechanical properties of SG-iron subjected to variable and isothermal austempering temperatures heat treatment. Materials Science and Engineering $A, \quad 340(1-2): 1-7$. http://dx.doi.org/10.1016/S0921-5093(02)00071-0

HANC, A.; BINCZYK, F. 2008. Structural analysis of austempered ductile iron obtained by Mössbauer spectroscopy. Archives of Materials Science and Engineering, 31(2):101-104

HSU, C.-H.; CHUANG, T.-L. 2001. Influence of stepped austempering process on the fracture toughness of austempered ductile iron. Metallurgical and Materials Transactions $A$, 32(10):2509-2514. http://dx.doi.org/10.1007/s11661-001-0040-y

HUPALO, M.F.; BALZER, M.E.; WARMLING, G. 2006. Avaliação da austemperabilidade de um ferro fundido nodular da classe perlítica (FE-70002) em corpos de prova com 50 mm de espessura. In: CBECIMat - 
CONGRESSO BRASILEIRO DE ENGENHARIA E CIÊNCIA DOS MATERIAIS, 17, Foz do Iguaçu, 2006. Anais... Foz do Iguaçu, p.7334-7345

NOFAL A.A.; JEKOVA L. 2009. Novel processing techniques and applications of austempered ductile iron. Journal of the University of Chemical Technology and Metallurgy, 44(3):213-228.

PUTATUNDA, S.K. 2001. Development of austempered ductile cast iron (ADI) with simultaneous high yield strength and fracture toughness by a novel two-step austempering process. Materials Science and Engineering A, 315(1-2):70-80. http://dx.doi.org/10.1016/S0921-5093(01)01210-2

RAVISHANKAR K.S.; RAJENDRA UDUPA, K.; PRASAD RAO, P. 2008. Development of austempered ductile iron for high tensile and fracture toughness by two step austempering process. In: WFC - WORLD FOUNDRY CONGRESS, 68, Cheenai, 2008. Anais... Cheenai, p. 35-40.

SANTOS, A.B. de S.; BRANCO, C.H.C. 1989. Metalurgia dos ferros fundidos cinzentos e nodulares. São Paulo, Instituto de Pesquisas Tecnológicas - IPT, $241 \mathrm{p}$.

TRUDEL, A.; GAGNÉ, M. 1997. Effect of composition and heat treatment parameters on the characteristics of austempered ductile irons. Canadian Metallurgical Quarterly, 36(5):289-298. http://dx.doi.org/10.1016/S0008-4433(97)00028-1

VALDES, C.; PEREZ LOPEZ, M.J.; FIGUEROA, M.; RAMIREZ, L.E. 2009. Austempered ductile iron with dual matrix structures. Revista Mexicana de Física, 55(1):48-51.

YESCAS-GONZÁLEZ, M.A. 2001. Modelling the microstructure and mechanical properties of austempered ductile irons. Disponível em: http://www.msm.cam.ac.uk/phase-trans/2000/phd.html\#miguel. Acesso em: 20/12/2003. 